\title{
3 Research Square \\ First Experience of Standard Linac, Rapid Arc and Dose-drop Scheme Based Total Body Irradiation
}

\section{Zhiyuan Xu}

University of Hong Kong-Shenzhen Hospital https://orcid.org/0000-0001-9663-8401

\section{Li Yang}

Radiation oncology

\section{Tim Hui}

Radiation oncology

\section{Xiaoqin Jiang}

Radiation oncology

Jeff chan

Radiation oncology

Jacob Cheung

Radiation oncology

Qian Wang

Radiation oncology

\section{Eric Lee}

Radiation oncology

Cheng Zhou

Radiation oncology

\section{Linbo Cai}

Radiation oncology

Longhua Chen ( $\nabla$ chenlhsum@126.com )

Radiation oncologist, Department of Radiation Oncology, Nanfang Hospital, Southern Medical University, Guangzhou 510515, China.

\section{Research}

Keywords: total body irradiation, rapid arc, positioning related dose deviation, quality assurance

Posted Date: November 9th, 2020

DOI: https://doi.org/10.21203/rs.3.rs-102601/v1

License: (1) (1) This work is licensed under a Creative Commons Attribution 4.0 International License. Read Full License 
Page $2 / 17$ 


\section{Abstract}

Background and purpose: To introduce a standard linac with true attachment free, rapid arc and dosedrop scheme for positioning related dose deviation control based total body irradiation(TBI).

Materials/Methods: One eight years old girl diagnosed with acute lymphocytic leukemia underwent TBI in 2020. Target volumes and organs at risk were contoured after CT simulation. Total sixteen ARC and four AP-PA from five isocenters were designed. A dose-drop scheme on both sides of adjacent region were performed to reduce positioning-related dose deviation. A series of quality assurance before radiotherapy and real-time dose monitoring during radiotherapy were carried out.

Results: The average on board imaging (OBI) time of per fraction was $40.3 \mathrm{~min}$, the average beam on time of per fraction was $37.2 \mathrm{~min}$, the average time to change from head first to feet first position was $18.4 \mathrm{~min}$. The average mean lung dose was $9.89 \mathrm{~Gy}$, the maximum lens dose was $7.60 \mathrm{~Gy}$, the mean PTV_total dose was $12.17 \mathrm{~Gy}, 98.23 \%$ PTV_total volume was covered by $90 \%$ of the prescription dose. The maximum dose (Dmax) of PTV_total was 13.65Gy. Dmean and V10.8 of PTV_total are only slightly different $(0.49 \%-1.89 \%$ and $0.26 \%-1.04 \%$ respectively) even with an error of $5-20 \mathrm{~mm}$ longitudinal misalignments. Gamma passing rate( $3 \mathrm{~mm} / 3 \%$ Gamma criteria) are between $93.5 \%$ and $100 \%$. Real-time dose monitoring showed an overall deviation of $-3.9 \% \pm 5.51 \%$.

Conclusions: Standard linac, rapid arc and dose-drop for positioning-related dose deviation control based total body irradiation is feasible, accurate, and reliable. It is worthy of clinical application.

\section{Introduction}

${ }^{1-3} \mathrm{TBI}$ is frequently used as a component of hematopoietic stem cell transplant (HSCT). ${ }^{4}$ The techniques of TBI vary widely from institution to institution. Conventional TBI often use two or three dimensional radiotherapy techniques, for example opposing anterior and posterior fields or lateral fields.

Disadvantages of conventional TBI include: 1. attachment devices and long source skin distance needed. 2. shielding specific organs are not precise. 3. poor dose uniformity. The inverse-planned intensitymodulated radiation therapy(IMRT) based TBI technique was developed to improve these shortcomings. But many IMRT TBI studies also need some special attachment and rare positioning related dose deviation control techniques. Now we report a standard linac with true attachment free, rapid arc and dose-drop positioning related dose deviation control based total body irradiation.

\section{Materials And Methods}

One eight years old girl diagnosed with acute lymphocytic leukemia underwent allogenic stem-cell transplantation following TBI and VP16 chemotherapy treatment at Hongkong university Shenzhen hospital in 2020. Patient was informed about the treatment and it's possible adverse events and about necessary diagnostics prior to treatment. Written consent by the patient herself and parents was obtained. 
Simulation occurs on a computed tomography scanner (Philip Brilliance CT Bigbore) with a setting of $120 \mathrm{kVp}, 3 \mathrm{~mm}$ slice. The scan length is limited to 1.15 meters. Both upper limbs are placed at the bilateral sides of the body. Head \& neck and shoulders thermoplastic mask, head rest and moldable head and neck support are used to fix the head and neck and shoulders. Whole body thermoplastic cast and whole body evacuated vacuum bags are used to fix thorax, abdomen, and limbs. The patient was positioned on a total body base board, in order to connect all above positioning devices as a whole unit as shown in Fig. 1. Prior to CT acquisition, radiopaque markers are placed near the umbilicus to serve as origin(Fig. 2) and a merge point of the two scans. The overlap area of two scanned images should be longer than $7 \mathrm{~cm}$ (5 cm for minimized dose gradient at radiation fields junctions, $2 \mathrm{~cm}$ for scattered dose calculation at radiation fields edge). If the patient is taller than 1.15 meters, two scans will be performed, the first scan (upper body) goes from the top of the head to pelvis with head first position, the second scan (lower body) goes from the bottom of the feet to the pelvis with feet first position. Both scans include the above origin markers. To allow for quality assurance (QA) measurements during radiotherapy, total eight $0.5 \mathrm{~cm}$ bolus are also scanned along with the patient, eight metal oxide semi-conductor field effect transistor (MOSFET) will be placed under bolus for timely treatment dose monitoring as shown in Fig. 1.

\section{Contouring}

Body was contoured using search body automatic tool, then manually modified to include all the outer body contour.

Even with a well-positioned devices, given the movement of the chest wall and ribs, The planning target volume (PTV) was contoured using the outer body contour minus bilateral lungs (except $3 \mathrm{~mm}$ margin of lung tissue adjacent to the ribs and chest wall to ensure full dose coverage of the ribs and chest wall). PTV_upper and PTV_lower were contoured on upper body and lower body scans respectively. PTV_total is equal to PTV_upper plus PTV_lower. PTV_crop was equal to PTV_total shrinks by $3 \mathrm{~mm}$ in the threedimensional direction. The right, left and bilateral lungs were contoured using the pulmonary windows separately according to RTOG atlases for Organs at Risk (OARs) in Thoracic Radiation Therapy.

Due to protection of lung tissues and dose coverage of chest wall, helping structures PTV_chestwall_1 cm was defined as the extrapulmonary three-dimensional area within $1 \mathrm{~cm}$. In order to ensure adequate skin dose coverage, the area with PTV_upper and PTV_lower expansion of $3 \mathrm{~mm}$ were named PTV_upper_3 $\mathrm{mm}$ and PTV_lower_3 $\mathrm{mm}$ respectively. At the same time, in order to reduce the actual dose coverage error caused by the positioning error, five sections of dose-drop transition zone were drawn continuously at the junction, the length of each section was $1 \mathrm{~cm}$, five sections were named as Step_12Gy-10Gy, Step_10Gy-8Gy, Step_8Gy-6Gy, Step_6Gy-4Gy and Step_4Gy-2Gy respectively as shown in Fig. 2. The parts of PTV_total in the dose-drop region was named PTV_drop. However, for dose statistics and dose volume histograms $(\mathrm{DVH})$, the anatomical lungs are the relevant structures. The right, left and bilateral kidneys were contoured if pre-existing renal insufficiency. Additional helping structures within the overlapping regions were contoured and used for steering the optimizer leading to an improved 
dose distribution in those areas. Other OARs were not routinely contoured and involved in dose optimization.

\section{Treatment planning and irradiation}

The full-body CT scan was imported into Eclipse treatment planning system, version 15.0. Isocenter was created for treatment planning of each scan. The total prescription dose was $12 \mathrm{~Gy}, 2 \mathrm{~Gy}$ per fraction, two fractions per day, the minimum interval was $6 \mathrm{~h}$. Irradiation was delivered at linac (Triology, Varian) based photon energy of $6 \mathrm{MV}$ over three consecutive days. The elevator and linear accelerator room were routinely disinfected before each fraction radiotherapy. The dose constraint is as follows: The minimum dose (Dmin) of PTV is more than or equal to $90 \%$ of prescription dose; The Dmax of PTV is less than or equal to $130 \%$ of prescription dose; The volume of PTV covered by $120 \%$ of the prescription dose should be less than $10 \%$. The mean dose of both lungs is less than or equal to $10 \mathrm{~Gy}$; The mean dose of both kidneys is less than or equal to $10 \mathrm{~Gy}$ if renal insufficiency, no special dose limitation for both kidneys if normal renal function.

As shown in Fig. 2, total sixteen ARC and four AP-PA from five isocenters were designed. For upper body treatment planning, total 12 ARCs were designed with three iso-centers because of the collimator field limitation is $40 \mathrm{~cm} \mathrm{X} 40 \mathrm{~cm}$. For lower body treatment planning, four AP-PA fields in fields were designed for bilateral shanks with a isocenter firstly. Then, another four ARCs with another isocenter were used to cover other parts of lower body. For the convenience of positioning, the coordinate values of each isocenter point are only different in the longitudinal direction. The connection between each rapid arc plan at each isocentric point was administrated by the function module of Base Dose Plan Compensation (BDPC).

Regarding the adjacent region dose distribution between upper and lower body radiotherapy, slight errors may cause significant hot and cold spots if conventional radiotherapy plans. Therefore, we designed a dose-drop scheme on both sides of adjacent region, which decreased from the prescribed dose (12 Gy) to 2 Gy within a length of $5 \mathrm{~cm}$.

We can treat upper body first with head first position, but re-position was needed with feet first position due to the limitation of the length of the linear accelerator treatment bed. For image guidance, kilo-voltage on broad imaging was used to collect images at anterior and right lateral directions for head and neck, abdomen, pelvis and lower limbs, and right lateral oblique directions for thorax to avoid obstruction by the arms. Online matching of the images with digital reconstruction radiograph (DRR) from the planning CTs were performed. Radiotherapy was permitted only if the senior physician and senior therapist confirm that the position error in each direction was less than $2 \mathrm{~mm}$. Treatment team will monitor the whole process with audio and video.

Quality assurance 
Before radiotherapy, dose verification was performed on each isocentric rapid arc or AP-PA plan and each radiation fields using ScandiDos Delta4PT 3D QA phantom. PTW Octavius 4D QA phantom was used for Gamma passing rate (3 mm/3\% Gamma criteria) at intersection of each two isocentric rapid arc plans of upper body. Point dose deviation at bilateral lower limbs and the junction of upper body and lower body were verified by a $60 \mathrm{~cm}$ (length) X 30cm(width) X $10 \mathrm{~cm}$ (height) solid water phantom, PinPoint $0.015 \mathrm{cc}$ and UNDOSE electrometer. During the process of each fraction radiotherapy, total eight interest point sites dose monitoring were needed using MOSFET. Points of interest include forehead, bilateral chest, navel, perineum, bilateral knee and unilateral foot.

\section{Results}

$\mathrm{OBI}$ time of per fraction was $40.3 \mathrm{~min}$, the average beam on time of per fraction was $37.2 \mathrm{~min}$, the average time to change from head first to feet first position was $18.4 \mathrm{~min}$. The average mean lung dose was $9.89 \mathrm{~Gy}$, the maximum lens dose was $7.60 \mathrm{~Gy}$, the mean PTV_total dose was $12.17 \mathrm{~Gy}, 98.23 \%$ PTV_total volume was covered by $90 \%$ of the prescription dose. The Dmax of PTV_total was $13.65 \mathrm{~Gy}$. The mean PTV_crop dose was $12.23 \mathrm{~Gy}$, $99.38 \%$ PTV_crop volume was covered by $90 \%$ of the prescription dose, the Dmax of PTV_crop was $13.65 \mathrm{~Gy}$. Dose distribution of TBI was shown in Fig. 2.

The mean PTV_drop dose was $12.13 \mathrm{~Gy}, 98.29 \%$ PTV_drop volume was covered by $90 \%$ of the prescription dose. The Dmax of PTV_drop was $13.31 \mathrm{~Gy}$. The mean PTV_drop_crop dose was $12.17 \mathrm{~Gy}$, 99.54\% PTV_drop_crop volume was covered by $90 \%$ of the prescription dose. The Dmax of PTV_drop_crop was $13.31 \mathrm{~Gy}$.

When plans were recalculated with longitudinal misalignments, DVH parameters of PTV_total and PTV_crop such as Dmax and Dmax difference(\%), Dmean and Dmean difference(\%), V10.8 and V10.8 difference(\%) were obtained respectively. Table 1 showed that Dmean and V10.8 are only slightly different even with an error of 5-20 mm longitudinal misalignments. Considering that the positioning error of $\mathrm{OBI}$ verification can be controlled smaller than $2-5 \mathrm{~mm}$, the change of Dmax is also acceptable. 
Table 1

Dosimetry parameter if an error of 5-20 mm closer longitudinal misalignments

\begin{tabular}{|llllllll|}
\hline Table 1 & & Dmax & $\begin{array}{l}\text { Dmax } \\
\text { difference(\%) }\end{array}$ & Dmean & $\begin{array}{l}\text { Dmean } \\
\text { difference(\%) }\end{array}$ & V10.8 & $\begin{array}{l}\text { V10.8 } \\
\text { difference(\%) }\end{array}$ \\
\hline No shift & PTV_total & 13.65 & 0.00 & 12.17 & 0.00 & 98.24 & 0.00 \\
\hline $\begin{array}{l}5 \mathrm{~mm} \\
\text { closer }\end{array}$ & PTV_crop & 13.65 & 0.00 & 12.23 & 0.00 & 99.37 & 0.00 \\
\hline & PTV_crop & 16.79 & 23.00 & 12.30 & 0.57 & 97.98 & -0.26 \\
\hline $\begin{array}{l}10 \mathrm{~mm} \\
\text { closer }\end{array}$ & PTV_total & 19.83 & 45.27 & 12.27 & 0.82 & 97.24 & -0.13 \\
\hline & PTV_crop & 19.83 & 45.27 & 12.35 & 0.98 & -0.96 \\
\hline $\begin{array}{l}15 \mathrm{~mm} \\
\text { closer }\end{array}$ & PTV_total & 20.90 & 53.11 & 12.34 & 1.40 & 98.65 & -0.72 \\
\hline & PTV_crop & 20.90 & 53.11 & 12.42 & 1.55 & 97.31 & -0.95 \\
\hline $\begin{array}{l}20 \mathrm{~mm} \\
\text { closer }\end{array}$ & PTV_total & 20.93 & 53.33 & 12.40 & 1.89 & 97.66 & -0.71 \\
\hline & PTV_crop & 20.93 & 53.33 & 12.48 & 2.04 & -1.04 \\
\hline
\end{tabular}

Table 2 showed dose verification of each isocentric rapid arc or AP-PA plan and each radiation fields were in compliance with the requirements perfectly. Gamma passing rates ( $3 \mathrm{~mm} / 3 \%$ Gamma criteria) at intersection of each two isocentric plans of upper body were $98.4 \%$ and $95.9 \%$ respectively, the standard requirements have been well passed. Point dose deviation at bilateral lower limbs and the junction of upper body and lower body were from $1.5-3.38 \%$, the values were also meet the standard requirements. 
Table 2

Dose verification of each isocentric rapid arc or AP-PA plan and each radiation field

\begin{tabular}{|c|c|c|c|c|}
\hline & Isocenter & $\begin{array}{l}\text { Gamma passing rate } \\
\text { (3 mm/ 3\% Gamma criteria) }\end{array}$ & Fields & $\begin{array}{l}\text { Gamma passing rate } \\
\text { (3 mm/ 3\% Gamma criteria) }\end{array}$ \\
\hline \multirow[t]{12}{*}{ Upper body } & Isocenter 1 & \multirow[t]{4}{*}{$100 \%$} & 1 & $100 \%$ \\
\hline & \multirow[t]{3}{*}{ Fields $1-4$} & & 2 & $100 \%$ \\
\hline & & & 3 & $100 \%$ \\
\hline & & & 4 & $100 \%$ \\
\hline & Isocenter 2 & \multirow[t]{4}{*}{$99.3 \%$} & 5 & $99.9 \%$ \\
\hline & \multirow[t]{3}{*}{ Fields 5-8 } & & 6 & $100 \%$ \\
\hline & & & 7 & $99.4 \%$ \\
\hline & & & 8 & $100 \%$ \\
\hline & Isocenter 3 & \multirow[t]{4}{*}{$95.3 \%$} & 9 & $99.8 \%$ \\
\hline & \multirow[t]{3}{*}{ Fields 9-12 } & & 10 & $100 \%$ \\
\hline & & & 11 & $99.4 \%$ \\
\hline & & & 12 & $100 \%$ \\
\hline \multirow[t]{8}{*}{ Lower body } & Isocenter 4 & $93.5 \%$ & 13 & $99.8 \%$ \\
\hline & \multirow[t]{3}{*}{ Fields $13-16$} & & 14 & $100 \%$ \\
\hline & & & 15 & $100 \%$ \\
\hline & & & 16 & $100 \%$ \\
\hline & Isocenter 5 & $95.2 \%$ & 17 & $100.0 \%$ \\
\hline & \multirow[t]{3}{*}{ Fields 17-20 } & & 18 & $99.1 \%$ \\
\hline & & & 19 & $100 \%$ \\
\hline & & & 20 & $98.4 \%$ \\
\hline
\end{tabular}

During the process of each fraction radiotherapy, total eight interest point sites dose monitoring were done, then all the isocentric accumulative doses were added and analyzed, the results showed an overall deviation of $-3.9 \% \pm 5.51 \%$.

\section{Discussion}


5,6 TBI is mainly used for leukemia, followed by malignant lymphoma, myelodysplastic syndrome, multiple myeloma and other malignant diseases. It is also used for benign diseases such as aplastic anemia. ${ }^{7-11}$ Outcomes after fractionated TBI were superior as compared with chemotherapy based conditioning with regard to overall survival, leukemia-free survival, relapse incidence, and non-relapse mortality. TBI in conjunction with chemotherapeutic agents has proven useful for eradicating residual malignant cells and for immunosuppression before HSCT. Unique features of TBI that make it a valuable component of transplant preparative regimens include: 1. No sparing of "sanctuary" sites such as testes and the central nervous system. 2. Dose homogeneity to the whole body regardless of blood supply. 3 . Less chance of cross-resistance with other antineoplastic agents (chemotherapy). 4. No problems with excretion or detoxifification. 5. Ability to tailor the dose distribution by shielding specific organs or by "boosting" sites. It is essential that the complicated treatment and care of the patient receiving TBI be well coordinated among the various subspecialties (medical oncology, radiation oncology, etc.) and caregivers (physicians, nurses, physicists, therapist psychologists, dieticians, etc).

${ }^{12}$ Most centers use opposing anterior and posterior fields with the patient standing upright several meters from the source and the beam pointed horizontally, thickness variations have less effect on dose homogeneity for This approach; however, the typical standing positioning can be strenuous with poor tolerance. ${ }^{13}$ Patients also can be irradiated with lateral fields in a sitting or partly reclining position with better tolerance, but variations in patient thickness can cause large dose heterogeneities, especially if compensators are not utilized during treatment. Most importantly, these above conventional large-field techniques require an extended source-to skin distance (SSD) that may not be available in standard-sized Linear accelerator room. ${ }^{14}$ Petra M. Härt et al introduced a sweeping beam technique for total body irradiation in standard treatment rooms and for standard linear accelerators. The patient is positioned on a low couch on the floor, the longitudinal axis of the body in the rotational plane of the linac. The sweeping beam technique needs the couch on the floor with the Makrolon plate on top. The inverseplanned IMRT based TBI technique was developed to improve upon these shortcomings, ${ }^{15,16}$ a benefit could be demonstrated with regard to dose distribution and homogeneity and the selective dose-reduction to organs at risk.

17 Patients taller than $120 \mathrm{~cm}$ cannot be treated in one position due to the limited cranial-caudal couch shift capacities of the linac. Therefore, patients are usually turned from a head-first supine position (HFS) to a feet-first supine position (FFS) to overcome this limitation. Losert $C$ et al showed a newly developed rotatable tabletop consists completely of carbon fiber, including the ball bearing within the base plate of the rotation unit. The patient can be turned $180^{\circ}$ from a HFS to a FFS position within a few seconds, without the need of repositioning. ${ }^{18}$ Treatment plans with an indexed rotational immobilization system had multi-isocentric volumetric modulated arc therapy (VMAT) beams to the upper body and parallel opposed fields to the lower body, with a 12 Gy prescription dose to $>90 \%$ of the body and mean lung dose $\sim 8 \mathrm{~Gy}$. In the end-to-end test, point dose measurements had $<10 \%$ error. Compared to conventional TBI, the VMAT-based TBI technique increased the mean dose to the body by $\sim 1.0-1.5 \mathrm{~Gy}$ and decreased the mean dose to the lung by $\sim 1.0-1.5 \mathrm{~Gy}$. The main problem of this approach is additional use of the 
special rotatable tabletop at the linear accelerator, it is not available in most hospital around the world.

${ }^{16}$ Bora Tas et al introduced total-body irradiation using linac-based volumetric modulated arc therapy, high-dose junction regions were eliminated after the registration of two CT sets via bias-dose properties of Monaco 5.11 TPS, Plan adaptation delivery while ensuring OAR tolerances never exceeded due to biasdose planning because TPS considers dose distribution from previous plans while optimizing the cumulative dose distribution, but when plans were recalculated with each $3.0-\mathrm{mm}, 6.0-\mathrm{mm}, 9.0-\mathrm{mm}$, and 12.0-mm longitudinal misalignments, an average of $2.0 \% \pm 0.7,3.7 \% \pm 1.2,6.5 \% \pm 1.7$ and $7.2 \% \pm 1.7$ higher mean lung doses; $2.6 \% \pm 1.5,9.9 \% \pm 3.7,15.1 \% \pm 4.2$ and $22.6 \% \pm 3.1$ higher maximum lung doses and $3.6 \% \pm 1.3,11.9 \% \pm 3.2,20.4 \% \pm 3.6$ and $22.0 \% \pm 3.8$ higher point doses were obtained respectively. Our studies showed dose-drop positioning related dose deviation control technology can reduce dose distribution error.

${ }^{19}$ Naoya Ishibashi showed fractionated total body irradiation with myeloablative conditioning given at a lower dose (<12 Gy vs 12 Gy) did not decrease the incidence of adverse events. So the most common prescription dose is $12 \mathrm{~Gy} .{ }^{11,20} \mathrm{TBI}$ can cause severe late-effects such as growth deficiency, sclerodermic chronic graft vs. host disease, osteonecrosis, impaired fertility, diabetes, pulmonary late effects, chronic renal insufficiency, cardiac late-effects, subsequent cancers, blindness and so on. More and more studies focus on late side effects. Pulmonary late effect is one of the main side effects. ${ }^{21} \mathrm{Natia}$ Esiashvili et al showed patients treated with mean lung dose $<800 \mathrm{cGy}$ had better outcomes. Lateral fields were associated with higher lung dose, and thus they should be avoided.

\section{Conclusion}

Standard linac, rapid arc and dose-drop positioning error control based total body irradiation is safe and reliable. Advantages include: 1. a standard linac with true attachment free. 2. shielding specific organs and target volume dose coverage are precise by rapid arc. 3. excellent dose-drop scheme for positioning related dose deviation control. But good training in TBI-related techniques are needed first.

\section{Abbreviations}

$\mathrm{TBI}$, total body irradiation; $\mathrm{OBI}$, on board imaging; $\mathrm{AP}-\mathrm{PA}$, anterior posterior; Dmax, maximum dose; Dmean, the mean dose; PTV, planning target volume; Dmin, minimum dose; HSCT, hematopoietic stem cell transplant; MOSFET, eight metal oxide semi-conductor field effect transistor; QA, quality assurance; OAR, organs at risk; BDPC, Base Dose Plan Compensation; DVH, dose volume histograms; DRR, digital reconstruction radiograph; HFS, head-first supine position; FFS, feet-first supine position; VMAT, volumetric modulated arc therapy.

\section{Declarations}

Funding and Competing interests 
This project is supported in part by Health Commission of Guangdong Province, China (NO. B2020100). The authors declare that they have no other competing interests.

Ethics approval『consent to participate and consent for publication

All patients signed, at hospital admission, consent for the use of their data for retrospective and scientific investigation. The paper has been performed in accordance with the declaration of helsinki and has been approved by the local ethics committee.

Availability of data and material

Not applicable

Competing interests

No

Funding

This project is supported in part by Health Commission of Guangdong Province, China (NO. B2020100). No other conflict of interest exits in the submission of this manuscript, and manuscript is approved by all authors for publication. I would like to declare on behalf of my co-authors that the work described was original research that has not been published previously, and not under consideration for publication elsewhere, in whole or in part. All the authors listed have approved the manuscript that is enclosed.

Authors' contributions

Study design: Longhua Chen, Zhiyuan Xu, Li Yang. Literature research: Zhiyuan Xu, Li Yang. Data acquisition: Zhiyuan Xu, Li Yang, Tim Hui, Xiaoqin Jiang, Jacob Cheung, Jeff chan, Qian Wang, Eric Lee. Statistical analysis: Zhiyuan Xu, Li Yang, Tim Hui, Xiaoqin Jiang. Manuscript preparation: Zhiyuan Xu, Li Yang. Manuscript revision/review: Longhua Chen, Zhiyuan Xu, Li Yang, Tim Hui, Xiaoqin Jiang, Jacob Cheung.

Acknowledgements

Thanks to the pediatric oncologists, nurses, therapists, physicist and physicians who participated in this study.

\section{References}

1. Suzanne L Wolden, Rachel A Rabinovitch, Nathan H J Bittner, et al. American College of Radiology (ACR) and American Society for Radiation Oncology (ASTRO) practice guideline for the performance of total body irradiation (TBI). Am J Clin Oncol. 2013 Feb;36(1):97-101. doi:

10.1097/COC.0b013e31826e0528. 
2. Francesca DE Felice, Lavinia Grapulin, Daniela Musio,et al. Treatment Complications and Long-term Outcomes of Total Body Irradiation in Patients with Acute Lymphoblastic Leukemia: A Single Institute Experience. Anticancer Res. 2016 Sep;36(9):4859-64. doi: 10.21873/anticanres.11049.

3. Tracy L Gieger, Michael W Nolan, Donald M Roback,et al. Implementation of total body photon irradiation as part of an institutional bone marrow transplant program for the treatment of canine lymphoma and leukemias. Vet Radiol Ultrasound. 2019 Sep;60(5):586-593. doi:

10.1111/vru.12776. Epub 2019 May 30.

4. Van Dyk J, Galvin JM, Glasgow GP, et al. AAPM Report No. 17: the physical aspects of total and half body photon irradiation. American Association of Physicists in Medicine. June 1986. https://www.aapm.org/pubs/reports/RPT_17.pdf.

5. Hiroki Kawaguchi, Toshinori Soejima, Naoya Ishibashi,et al. National survey on total-body irradiation prior to reduced-intensity stem cell transplantation in Japan: The Japanese Radiation Oncology Study Group. J Radiat Res. 2019 Oct 23;60(5): 579-585. doi: 10.1093/jrr/rrz028.

6. Frank Heinzelmann, Helmut Ottinger, Carl-Heinz Müller,et al. Total-body irradiation--role and indications: results from the German Registry for Stem Cell Transplantation (DRST). Strahlenther Onkol. 2006 Apr;182(4):222-30. doi: 10.1007/s00066-006-1468-1.

7. Andre Manfred Willasch, Christina Peters, Petr Sedláček,et al. Myeloablative conditioning for alloHSCT in pediatric ALL: FTBI or chemotherapy?-A multicenter EBMT-PDWP study. Bone Marrow Transplant. 2020 Aug;55(8):1540-1551. doi: 10.1038/s41409-020-0854-0. Epub 2020 Mar 17.

8. Enric Carreras, Carlo Dufour, Mohamad Mohty, et al. The EBMT Handbook: Hematopoietic Stem Cell Transplantation and Cellular Therapies. European Society for Blood and Marrow Transplantation. 2019.

9. N Bunin, R Aplenc, N Kamani,et al. Randomized trial of busulfan vs total body irradiation containing conditioning regimens for children with acute lymphoblastic leukemia: a Pediatric Blood and Marrow Transplant Consortium study. Bone Marrow Transplant. 2003 Sep;32(6):543-8. doi: 10.1038/sj.bmt.1704198.

10. Mudit Dutta BS, Rahul Mhaskar MPH, Hany Elmariah,et al. Impact of TBI-Based Conditioning Regimen on Outcomes of Patients with Acute Lymphoblastic Leukemia Undergoing Allogeneic Hematopoietic Cell Transplant: Systematic Review and Meta-Analysis. Blood (2019) 134 (Supplement_1): 4773. doi.org/10.1182/blood-2019-131126.

11. Sarah J Stephens, Samantha Thomas, David A Rizzieri,et al. Myeloablative conditioning with total body irradiation for AML: Balancing survival and pulmonary toxicity. Adv Radiat Oncol. 2016 Jul 15;1(4):272-280. doi: 10.1016/j.adro.2016.07.001.eCollection Oct-Dec 2016.

12. Rui Yao, Damian Bernard, Julius Turian,et al. A simplified technique for delivering total body irradiation (TBI) with improved dose homogeneity. Med Phys. 2012 Apr;39(4):2239-48. doi: 10.1118/1.3697526.

13. Susanta K Hui, R K Das, Bruce Thomadsen,et al. CT-based analysis of dose homogeneity in total body irradiation using lateral beam. J Appl Clin Med Phys. Autumn 2004;5(4):71-9. doi: 
10.1120/jacmp.v5i4.1980. Epub 2004 Oct 1.

14. Petra M Härtl, Marius Treutwein, Matthias $G$ Hautmann,et al. Total body irradiation-an attachment free sweeping beam technique. Radiat Oncol. 2016 Jun 10;11:81. doi: 10.1186/s13014-016-0658-y.

15. Arne Gruen, Wolfram Ebell, Waldemar Wlodarczyk,et al. Total Body Irradiation (TBI) using Helical Tomotherapy in children and young adults undergoing stem cell transplantation. Radiat Oncol. 2013 Apr 15;8:92. doi: 10.1186/1748-717X-8-92.

16. Bora Tas, Ismail Faruk Durmus, Ayse Okumus,et al. Total-body irradiation using linac-based volumetric modulated arc therapy: Its clinical accuracy, feasibility and reliability. Radiother Oncol. 2018 Dec;129(3):527-533. doi: 10.1016/j.radonc.2018.08.005.Epub 2018 Aug 29.

17. Christoph Losert, Roel Shpani, Robert Kießling,et al. Novel rotatable tabletop for total-body irradiation using a linac-based VMAT technique. Radiat Oncol. 2019 Dec 30;14(1):244. doi: 10.1186/s13014019-1445-3.

18. Ouyang L, Folkerts $\mathrm{M}$, Zhang $\mathrm{Y}$, et al: Volumetric modulated arc therapy based total body irradiation: Workflow and clinical experience with an indexed rotational immobilization system. Physics and Imaging in Radiation Oncology. 2017 Nov 30; 4:22-25, doi.org/10.1016/j.phro.2017.11.002

19. Naoya Ishibashi, Toshiya Maebayashi, Takuya Aizawa,et al. Various Regimens of Total Body Irradiation for Hematopoietic Stem Cell Transplant. Exp Clin Transplant. 2016 Dec;14(6):670-675.

20. Fernand Freycon, Léonie Casagranda, Béatrice Trombert-Paviot,et al. The impact of severe lateeffects after $12 \mathrm{~Gy}$ fractionated total body irradiation and allogeneic stem cell transplantation for childhood leukemia (1988-2010). Pediatr Hematol Oncol. 2019 Mar;36(2):86-102. doi: 10.1080/08880018.2019.1591549.Epub 2019 Apr 12.

21. Natia Esiashvili, Xiaomin Lu, Ken Ulin,et al. Higher Reported Lung Dose Received During Total Body Irradiation for Allogeneic Hematopoietic Stem Cell Transplantation in Children With Acute Lymphoblastic Leukemia Is Associated With Inferior Survival: A Report from the Children's Oncology Group. Int J Radiat Oncol Biol Phys. 2019 Jul 1;104(3):513-521. doi: 10.1016/j.jirobp. 2019.02.034. Epub 2019 Feb 23.

\section{Figures}




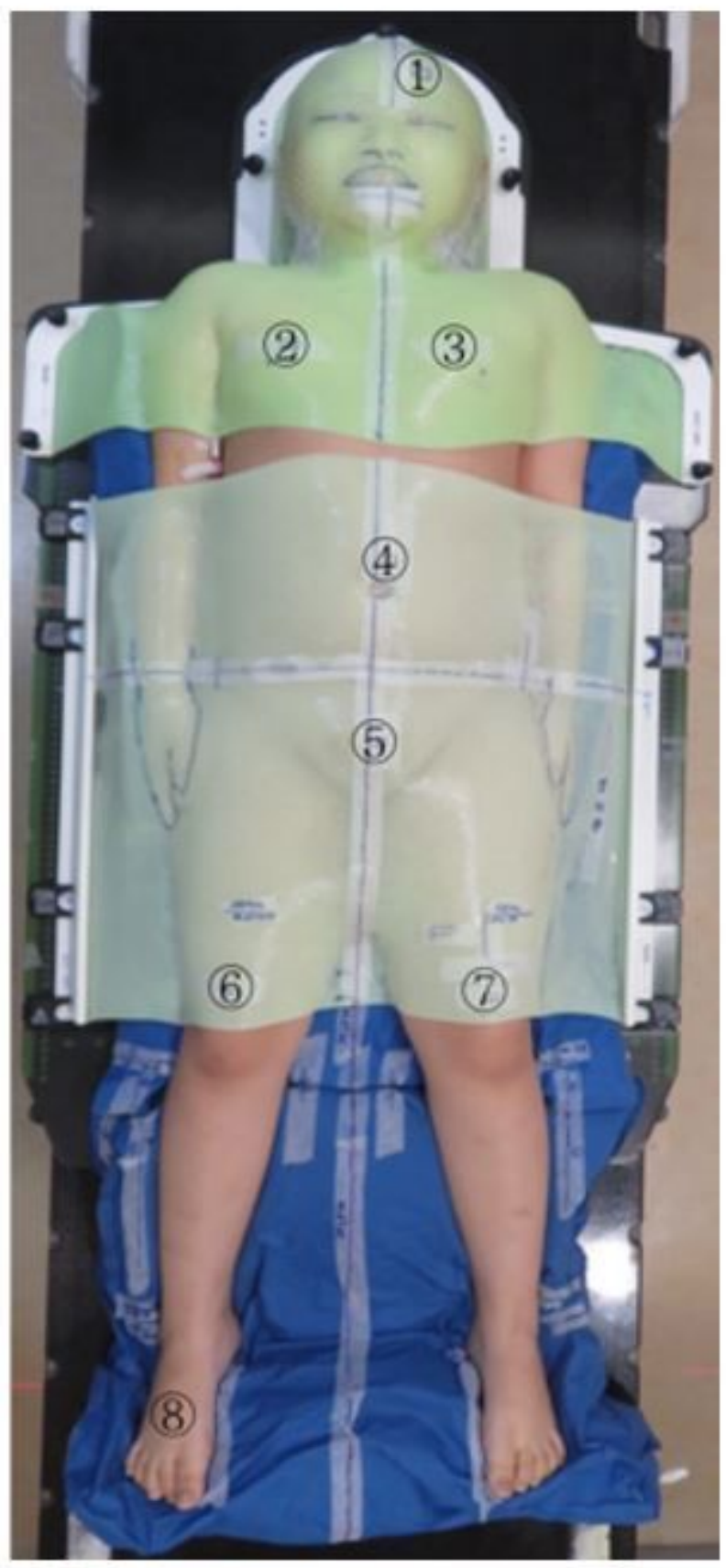

Figure 1

Positioning and fixation device, $\nabla-\nabla$ shows MOSFET position diagram 


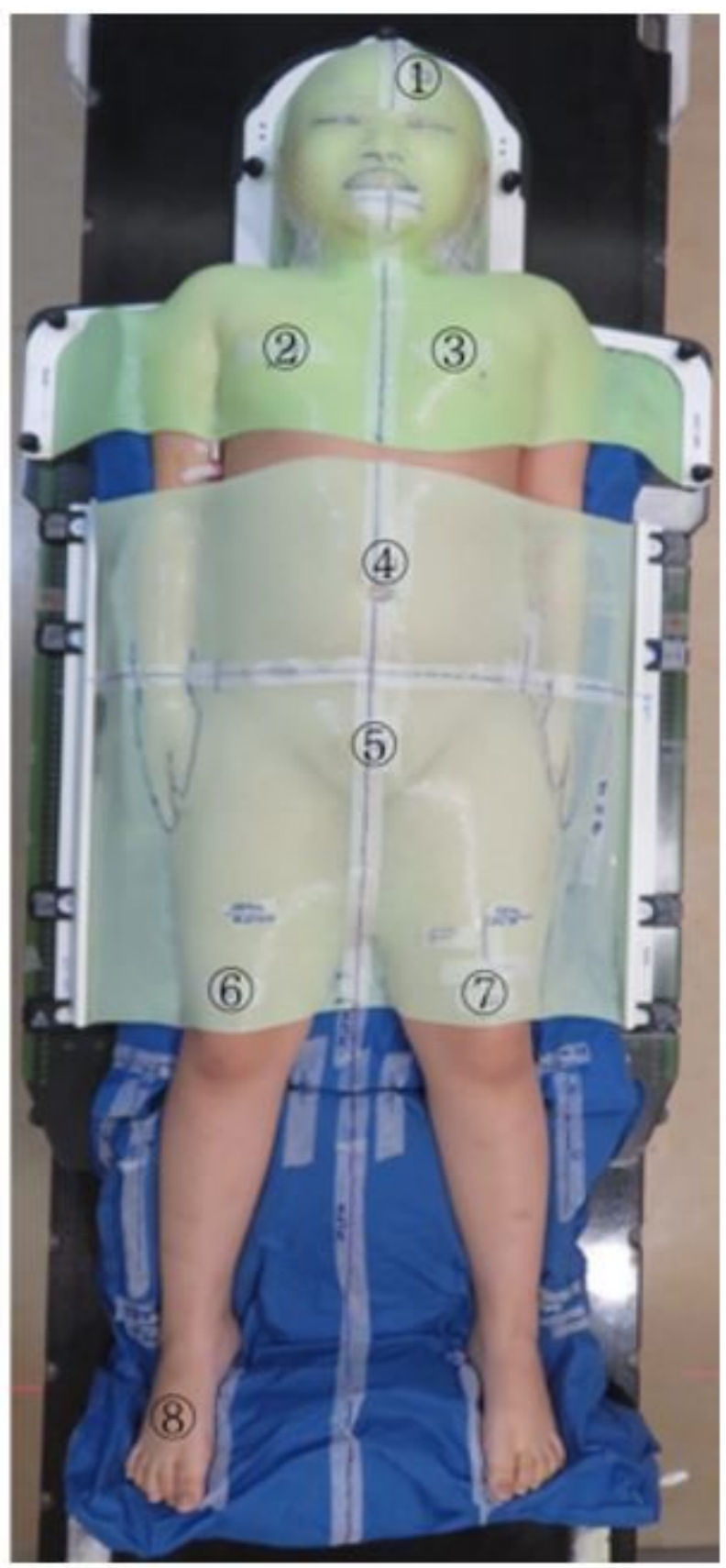

Figure 1

Positioning and fixation device, $\nabla-\nabla$ shows MOSFET position diagram 


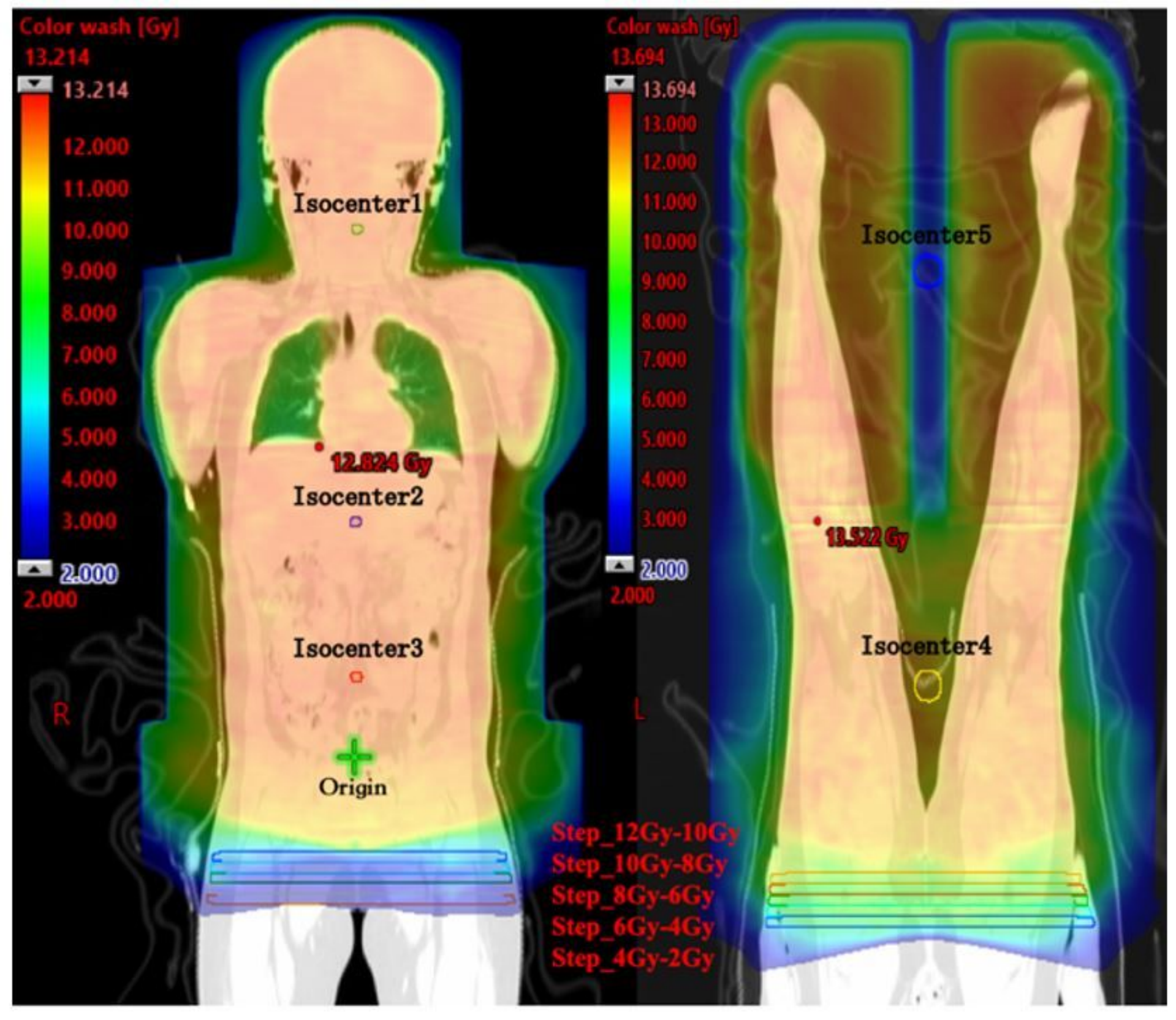

Figure 2

Dose distribution, five dose-drop sections and five isocenters. 


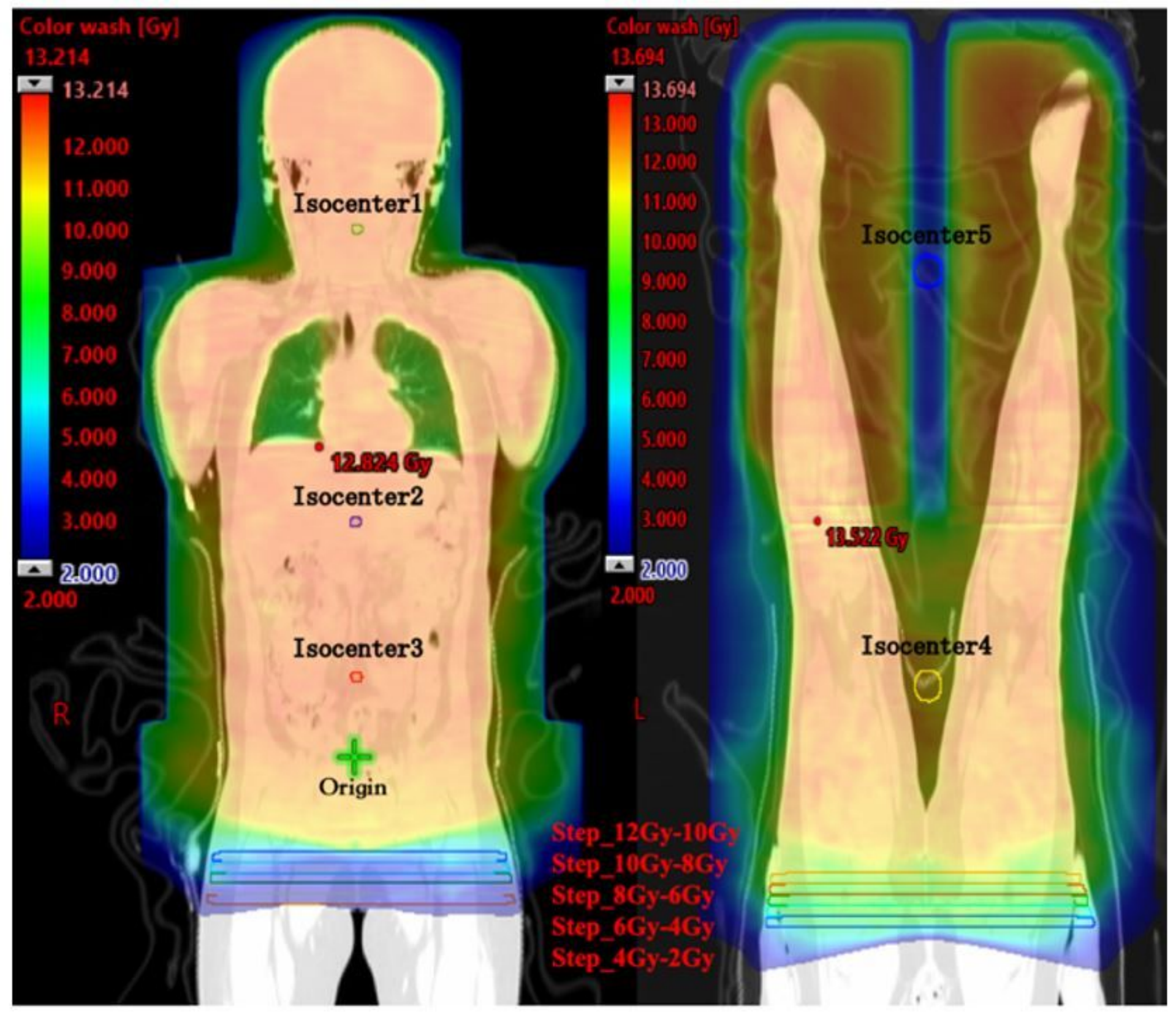

Figure 2

Dose distribution, five dose-drop sections and five isocenters. 